\title{
Contrast-Enhanced Dynamic Magnetic Resonance Imaging of Primary Pulmonary Leiomyosarcoma
}

\author{
Tomayoshi Hayashi, Tsutomu Tagawa, ${ }^{1}$ Kazuto Ashizawa, ${ }^{2}$ Kuniko Abe, \\ Naoe Kinoshita, Shin Tsutsui, ${ }^{2}$ Masanobu Anami and Takeshi Nagayasu ${ }^{1}$ \\ Department of Pathology, Nagasaki University Hospital, Nagasaki, Japan, \\ ${ }^{1}$ Department of First Surgery, and ${ }^{2}$ Department of Radiology, Nagasaki \\ University Graduate School of Biomedical Sciences, Nagasaki, Japan
}

Hayashi, T., Tagawa, T., Ashizawa, K., Abe, K., Kinoshita, N., Tsutsui, S., Anami, M. and Nagayasu, T. Contrast-Enhanced Dynamic Magnetic Resonance Imaging of Primary Pulmonary Leiomyosarcoma. Tohoku J. Exp. Med., 2006, 210 (3), 263-267 — An abnormal opacity was found incidentally on chest radiography in a 19-year-old Japanese man with lumbago. Chest computed tomography (CT) revealed a $55 \times 40 \mathrm{~mm}$ welldefined mass with smooth margins in the lower lobe of the right lung (S6). Contrastenhanced dynamic CT showed that the lesion was not enhanced, suggesting a cystic lesion, such as a bronchogenic cyst. However, contrast-enhanced dynamic magnetic resonance imaging (MRI) revealed enhancement of the lesion, which had a clear margin and homogenous consistency, suggesting a benign pulmonary solid tumor. Video-assisted thoracoscopic surgery, S6 segmentectomy, was performed, disclosing the lesion to be solid. Histopathologically, the tumor consists of spindle cells with ample eosinophilic cytoplasm arranged in an interlacing pattern. Immunohistochemical staining revealed positive desmin reactivity but was negative for $\alpha$ smooth muscle actin and HHF-35 (muscle specific actin), indicating atypical phenotypic differentiation. The lesion was accordingly diagnosed as leiomyosarcoma with low grade malignancy, and right lower lobectomy was performed. The patient has remained free from recurrence for 18 months without any additional chemotherapy or radiation therapy. Contrast-enhanced dynamic MRI should be carried out to facilitate accurate preoperative diagnosis and selection of the correct surgical plan. - pulmonary; leiomyosarcoma; contrast-enhanced dynamic CT; cyst; contrast-enhanced dynamic MRI

(C) 2006 Tohoku University Medical Press

Primary pulmonary leiomyosarcoma is a rare neoplasm reported to originate from pulmonary arteries, veins, or parenchyma (Ohta et al. 1992; Moran et al. 1997; Fitoz et al. 2000; Silvestris et al. 2003). It is further classified into bronchial and peripheral types by its location and origin
(Hartman and Shochat 1983; Shimota et al. 1991; Ohta et al. 1992; Attanoos et al. 1996; Moran et al. 1997; Silvestris et al. 2003).

In general, the radiological features of sarcomas are non-specific; these tumors appear expansive with distinct margins and are difficult to

Received June 20, 2006; revision accepted for publication September 6, 2006.

Correspondence: Tomayoshi Hayashi, M.D., Ph.D., Department of Pathology, Nagasaki University Hospital,

1-7-1 Sakamoto, Nagasaki 852-8501, Japan.

e-mail: toma@nagasaki-u.ac.jp 
differentiate from carcinoma. While it is also difficult to make histological classification from radiological findings, higher grade sarcomas often exhibit internal necrosis, indicative of their aggressive nature (Fitoz et al. 2000; Gladish et al. 2002).

We report a case of primary pulmonary sarcoma in which contrast-enhanced dynamic computed tomography (CT) suggested a cystic tumor, and contrast-enhanced dynamic magnetic resonance imaging (MRI) was useful in establishing the correct diagnosis of the solid tumor. We received approval from the ethical committee of Nagasaki University Hospital regarding publication of the present case.

\section{Case Report}

The patient was a 19-year-old Japanese man. An abnormal opacity was found incidentally on chest radiography (Fig. 1) during the further investigation of lumbago. Chest CT revealed a 55 $\times 40 \mathrm{~mm}$ well-defined mass with smooth margins in the lower lobe of the right lung (S6 area). The lesion had homogeneous internal characteristics

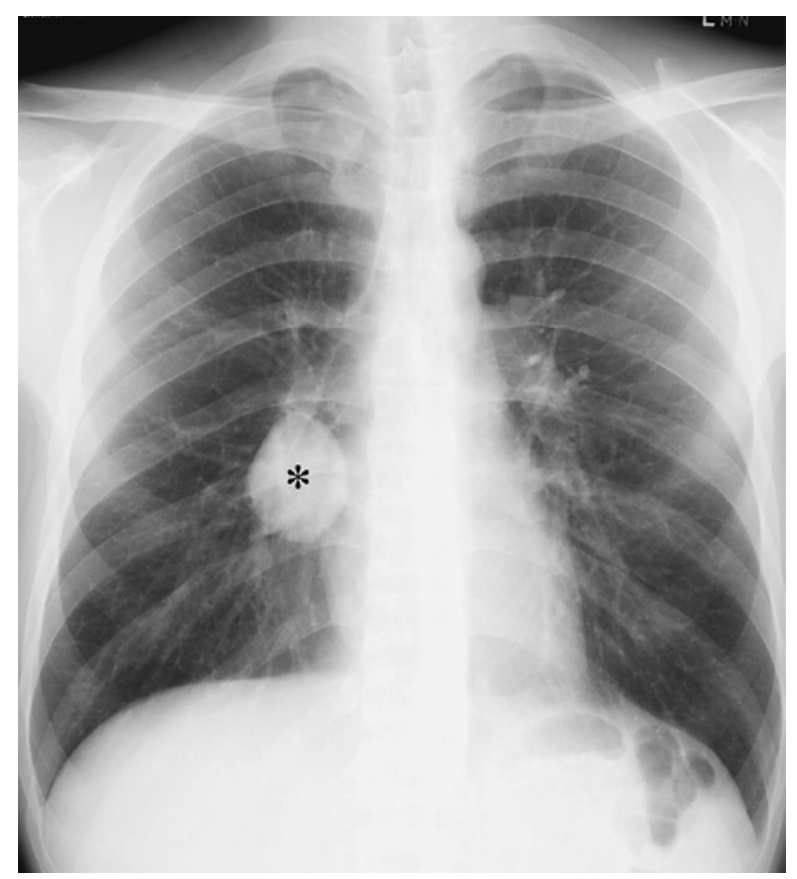

Fig. 1. Chest radiograph. An ellipsoid-shaped mass with smooth margins $(*)$ is seen in the infrahilar portion of the right lung. with a CT value of around 45 Hounsfield Units. Contrast-enhanced dynamic CT revealed no significant enhancement of the lesion, suggesting a cystic lesion such as bronchogenic cyst (Fig. 2). However, MRI revealed that the lesion was isointense on T1-weighted imaging and hyperintense on T2-weighted imaging compared to the intensity of surrounding muscles (Fig. 3a, b). These intensity findings did not suggest a cystic nature or myxoid degeneration of the lesion. In addition, contrast-enhanced dynamic MRI revealed gradual enhancement of the lesion, indicating a solid nature and suggesting a benign pulmonary solid tumor (Fig. 3c).

We subsequently performed video-assisted thoracoscopic surgery; S6 segmentectomy. Since intraoperative frozen section revealed benign tumor or low grade sarcoma, lower lobectomy was not performed.

The resected tumor was white-grey and solid with a distinct margin. We observed no necrosis or communication with large vessels or the main bronchus (Fig. 4a).

Histopathologically, the tumor was surrounded by fibrous capsule-like tissue consisting of compressed pulmonary parenchyma. Several bronchioles were visible at the periphery, indicating the origin of this lesion (Fig. 4b). The tumor consisted of spindle cells with ample eosinophilic

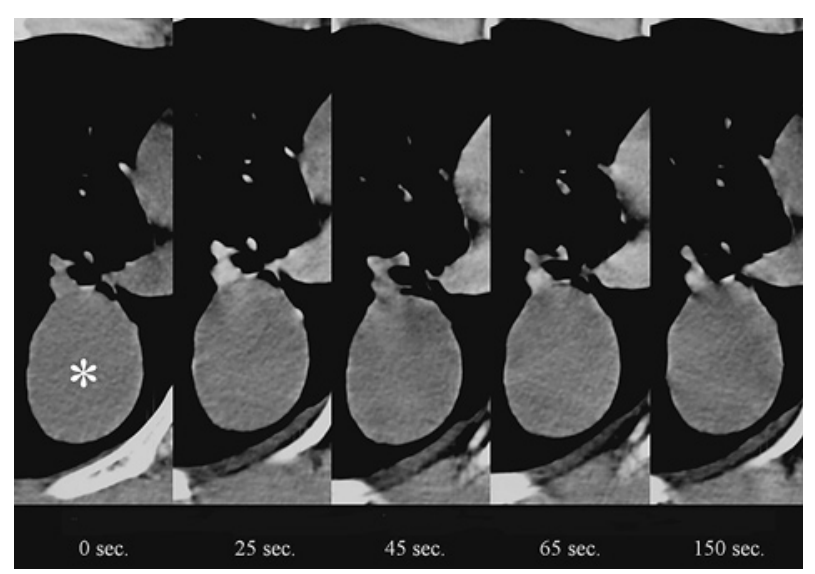

Fig. 2. Contrast-enhanced dynamic computed tomography images show no significant enhancement of the tumor $(*)$, indicating a cystic lesion such as bronchogenic cyst. 
cytoplasm arranged in an interlacing pattern (Fig. 4c). Scattered mitoses and mild nuclear atypia were evident (Fig. 4d). Immunohistochemically, tumor cells were positive for desmin (DAKO, Glostrup, Denmark) (Fig. 4e) and vimentin (DAKO), and negative for $\alpha$ smooth muscle (SM) actin (DAKO), HHF35 (muscle specific actin, DAKO), CD34 (Nichirei Biosciences, Tokyo), c-kit (Novocastra, Newcastle, UK), myoglobin (DAKO), HMB45 (melanoma antibody, DAKO), Melan-A (melanoma antibody, DAKO), CD21 (Novocastra), bcl-2 (DAKO), ALK (anaplastic lymphoma kinase, DAKO), AE1/AE3 (cytokeratin, DAKO), CAM5.2 (cytokeratin, Becton Dickinson, San Jose, CA, USA), and S-100 protein (DAKO) (Table 1).

The histopathological findings suggested a

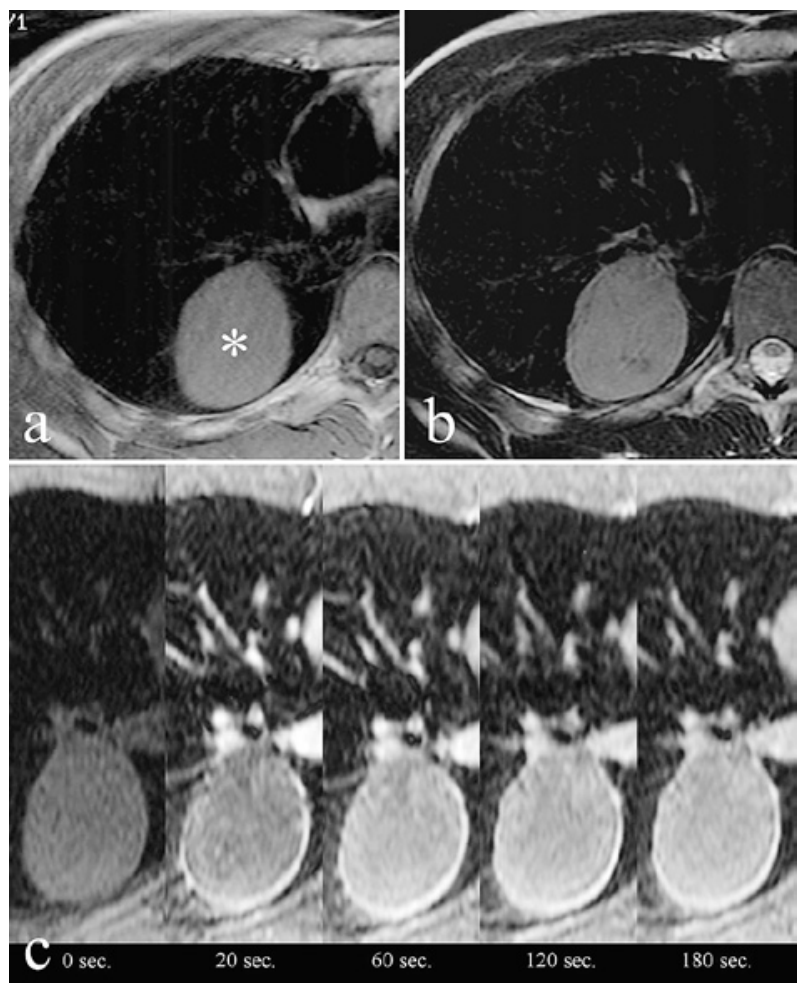

Fig. 3. Magnetic resonance imaging (MRI) revealed that the lesion $(*)$ was isointense on T1weighted imaging (a) and hyperintense on T2weighted imaging (b) compared to the intensity of surrounding muscles. Contrast-enhanced dynamic MRI revealed gradual enhancement of the lesion, indicating a solid nature and suggesting a benign pulmonary solid tumor (c). diagnosis of low grade leiomyosarcoma, peripheral type.

The patient accordingly underwent additional resection of the remnant right lower lobe, resulting in complete right lower lobectomy. Whole body examination by CT was performed and did not detect any other tumors. The patient

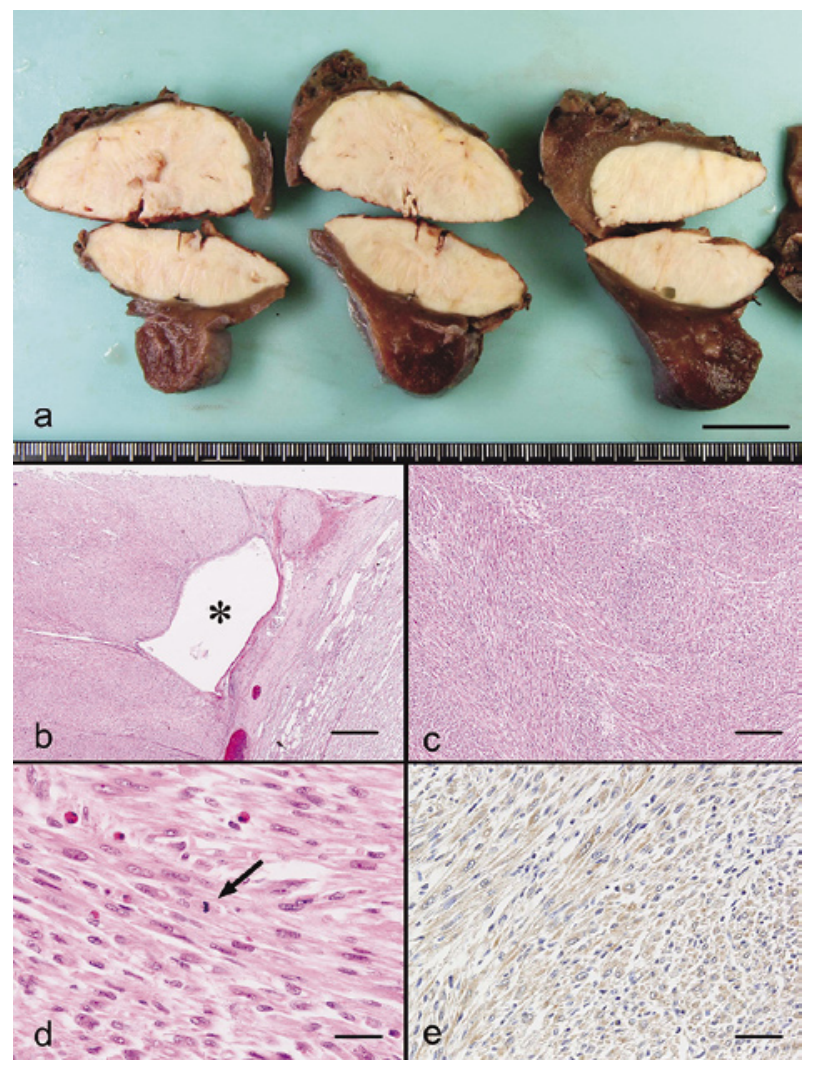

Fig. 4. Histopathological findings of the tumor.

a) Macroscopic photograph of the post-fixed cut surface of the tumor. The tumor is whitegray and solid, with no necrosis. No connections to large vessels or large bronchi are evident (bar $=2 \mathrm{~cm})$. b) Several bronchioles (*) are visible at the periphery of the tumor, indicating the origin of this lesion (Hematoxylin and eosin $[\mathrm{HE}], \times 20$, bar $=500 \mu \mathrm{m})$. c) The tumor consists of spindle cells with eosinophilic cytoplasm arranged in an interlacing pattern $(\mathrm{HE}, \times 40$, bar $=250 \mu \mathrm{m})$. d) At high magnification, spindle cells with mild nuclear atypia are arranged in a fascicular pattern. A mitosis is visible in this field (arrow) (HE, $\times$ 400 , bar $=25 \mu \mathrm{m})$. e) Immunohistochemically, the tumor cells are positive for desmin $(\times 200$, bar $=50 \mu \mathrm{m})$. 
TABLE 1. Details of primary antibodies used in this study.

\begin{tabular}{llcccc}
\hline Antigen & Catalog $\#$ & Source & Pretreatment $^{*}$ & Dilution & Result $^{-}$ \\
\hline Desmin & A0611 & DAKO & - & $1: 50$ & + \\
Vimentin & M7020 & DAKO & P & $1: 200$ & + \\
$\alpha$ smooth muscle actin & M0851 & DAKO & - & $1: 150$ & - \\
HHF35 (smooth muscle actin) & M0635 & DAKO & P & $1: 100$ & - \\
CD34 & NU-4A1 & Nichirei & - & Prediluted & - \\
& & biosciences & & & \\
c-kit & NCL-CD117 & Novocastra & P & $1: 20$ & - \\
Myoglobin & A0324 & DAKO & - & $1: 500$ & - \\
HMB45 (melanoma antiobdy) & M0634 & DAKO & P & $1: 50$ & - \\
Melan-A (melanoma antibody) & M7196 & DAKO & P & $1: 20$ & - \\
CD21 & NCL-CD21-2G9 & Novocastra & P & $1: 20$ & - \\
bcl-2 & M0887 & DAKO & P & $1: 20$ & - \\
ALK (anaplastic lymphoma kinase) & M7195 & DAKO & P & $1: 50$ & - \\
AE1/AE3 & M3515 & DAKO & Trypsin & $1: 100$ & - \\
CAM5.2 (cytokeratin) & 349205 & BECTON & Trypsin & Prediluted & - \\
& & DECKINSON & & & \\
S-100 & Z0311 & DAKO & - & $1: 700$ & - \\
\hline
\end{tabular}

${ }^{*} \mathrm{P}$, pressure cooker with pH6.0 citrate buffer.

is currently doing well and has been free from recurrence for 18 months postoperatively, without any additional chemotherapy or radiation therapy.

\section{Discussion}

Primary pulmonary leiomyosarcoma is extremely rare (Gladish et al. 2002). Although leiomyosarcoma is the most frequent type of primary pulmonary sarcoma (Janssen et al. 1994; Attanoos et al. 1996), immunohistochemical staining is essential for the correct classification of sarcomatous lesions (Moran et al. 1997).

In the present case, $\alpha \mathrm{SM}$ actin and HHF-35 reactivity, which are sensitive markers for smooth muscle, were absent, despite the fact that low grade sarcomas are generally better differentiated into normal smooth muscle cells. However, the literature does contain reports of pulmonary low grade leiomyosarcoma with negative $\alpha \mathrm{SM}$ actin and positive desmin reactivity (Moran et al. 1997). Staining with multiple antibodies such as $\alpha \mathrm{SM}$ actin, desmin, and HHF-35, is therefore necessary.

Leiomyosarcoma tends to occur in older patients; however, it has been reported in children and young patients, as in the present case (VeraRoman et al. 1983; Jimenez et al. 1986; Takeda et al. 2004).

Pulmonary leiomyosarcomas are subdivided into parenchymal, arterial, and venous types (Ohta et al. 1992; Moran et al. 1997; Fitoz et al. 2000). Since the present tumor had no connection to large vessels, it was classified as parenchymal type. This type is further subclassified into proximal bronchial and peripheral subtypes (Shimota et al. 1991; Ohta et al. 1992; Attanoos et al. 1996; Moran et al. 1997; Takakura et al. 2004). The present case belongs to the latter subtype, which is thought to originate from mesenchymal smooth muscle cells or smooth muscle of small bronchioles (Shimota et al. 1991; Ohta et al. 1992; Attanoos et al. 1996; Moran et al. 1997; Takakura et al. 2004).

In peripheral-type tumors such as the present case, prognosis depends on grade (Miller and Allew 1993; Janssen et al. 1994; Moran et al. 1997). Leiomyosarcomas are classified as low, intermediate, and high grade according to histo- 
logical findings. Low grade tumors do not tend to recur, and complete resection is therefore adequate. High grade tumors, however, have a high risk of metastasis and require postoperative chemotherapy (Moran et al. 1997). Good prognosis was anticipated for the present tumor, and indeed, no recurrence has occurred 18 months postoperatively.

Radiologically, sarcoma appears as a nonspecific solitary well-defined nodule/mass with smooth margins. Lobulation is not uncommon. Lesions often exhibit internal heterogeneity, indicating intratumoral hemorrhage and/or tumor necrosis (Fitoz et al. 2000; Gladish et al. 2002). However, tumors that have no necrosis and show no enhancement on contrast-enhanced CT are difficult to precisely differentiate from cystic lesions. Therefore, contrast-enhanced dynamic MRI can be useful in differentiating between cystic and solid lesions, particularly when it is not certain whether the lesion is solid on contrast-enhanced CT.

The histopathological differential diagnosis includes sarcomatoid/spindle cell neoplasms such as pleomorphic/spindle cell carcinoma, monophasic synovial sarcoma, solitary fibrous tumor, inflammatory pseudotumor, inflammatory myofibroblastic tumor, rhabdomyosarcoma, and follicular dendritic cell sarcoma, as well as leiomyosarcoma. However, the present histologic features such as distinct margins, interlacing patterns of rather monotone pleomorphic spindle cells, and lack of striation exclude these differential diagnoses, as does the panel of immunohistochemical markers that we used.

In conclusion, in this case of low grade primary pulmonary leiomyosarcoma, contrastenhanced dynamic CT findings were not particularly useful; however, contrast-enhanced dynamic MRI was useful in detecting a solid tumor, leading to resection and correct diagnosis utilizing a panel of immunohistochemical markers. We recommend contrast-enhanced dynamic MRI to facilitate accurate preoperative diagnosis and selection of the correct surgical plan.

\section{Acknowledgments}

The authors would like to express heartfelt thanks to Christopher D.M. Fletcher, M.D. FRCPath, Brigham \& Women's Hospital, Boston for his advice on the correct pathologic diagnosis.

\section{References}

Attanoos, R.L., Appleton, M.A. \& Gibbs, A.R. (1996) Primary sarcomas of the lung: a clinicopathological and immunohistochemical study of 14 cases. Histopathology, 29, 29-36.

Fitoz, S., Atasoy, C., Kizilkaya, E., Basekim, C. \& Karsli, F. (2000) Radiologic findings in primary pulmonary leiomyosarcoma. J. Thorac. Imaging, 15, 151-152.

Gladish, G.W., Sabliff, B.M., Munden, R.F., Truong, M.T., Erasmus, F.F. \& Chasen, M.H. (2002) Primary thoracic sarcomas. Radiographics, 22, 621-637.

Hartman, G.E. \& Shochat, S.J. (1983) Primary pulmonary neoplasms of childhood: a review. Ann. Thorac Surg., 36, 108-119.

Janssen, J.P., Mulder, J.J., Wagenaar, S.S., Elbers, H.R. \& van den Bosch, J.M. (1994) Primary sarcoma of the lung: a clinical study with long-term follow-up. Ann. Thorac. Surg., 58, 1151-1155.

Jimenez, J.F., Uthman, E.O., Townsend, J.W., Gloster, E.S. \& Seibert, J.J. (1986) Primary bronchopulmonary leiomyosarcoma in childhood. Arch. Pathol. Lab. Med., 110, 348-351.

Miller, D.L. \& Allen, M.S. (1993) Rare pulmonary neoplasms. Mayo Clin. Proc., 68, 492-498.

Moran, C.A., Suster, S., Abbondanzo, S.L. \& Koss, M.N. (1997) Primary leiomyosarcomas of the lung: a clinicopathologic and immunohistochemical study of 18 cases. Mod. Pathol, 10, 121-128.

Ohta, H., Komibuchi, T., Nishiyama, H., Shizuki, K. \& Miyaki, Y. (1992) 99mTc(V) -DMSA and 99mTc-MDP uptake and no $67 \mathrm{Ga}$-citrate uptake in a case of primary pulmonary leiomyosarcoma. Ann. Nucl. Med., 6, 191-193.

Shimota, H., Satoh, T., Ishi, K. \& Iwase, A. (1991) An autopsy case of primary lung leiomyosarcoma. Rinsho Byori, 39, 666-670. (in Japanese)

Silvestris, N., Piscitelli, D., Crucitta, E., Fiore, M., De Lena, M. \& Lorusso, V. (2003) Unusual response to second-line single-agent gemcitabine in locally advanced primary leiomyosarcoma of the lung: a case report. J. Chemother., 15, 507-509.

Takakura, R., Arita, K., Ohashi, N., Moritani, C., Nishino, R., Daga, H. \& Fujiwara, M. (2004) A case of primary pulmonary leiomyosarcoma with history of pneumonia 5 years before. Nihon Kokyuki Gakkai Zasshi, 42, 419-423. (in Japanese)

Takeda, F., Yamagiwa, I., Ohizumi, H. \& Shiono, S. (2004) Leiomyosarcoma of the main bronchus in a girl: a longtime survivor with multiple lung metastases. Pediatr. Pulmonol., 37, 368-374.

Vera-Roman, J.M., Sobonya, R.E., Gomez-Garcia, J.L., Sanz-Bondia, J.R. \& Paris-Romeu, F. (1983) Leiomyoma of the lung. Literature review and case report. Cancer, 52, 936-941. 